\title{
Kinematics and Internal Dynamics Formulations of the Canis Compressed Air Engine
}

\author{
Ngang Tangie Fru ${ }^{1}$ \\ ${ }^{1}$ Industrial Engineer-Manager \& Mechanical Engineer, IHSS (QHSE) Specialist \& Automobile Mechanics, Cameroon \\ Correspondence: Ngang Tangie Fru, Industrial Engineer-Manager \& Mechanical Engineer, IHSS (QHSE) Specialist \\ \& Automobile Mechanics, Cameroon. E-mail: tf.ngang@catuc.org
}

Received: November 15, 2016

Accepted: November 25, 2016

Online Published: May 31, 2017

doi:10.5539/mer.v7n1p44

URL: https://doi.org/10.5539/mer.v7n1p44

\begin{abstract}
Prior to this paper some study was conducted that resulted to the conceptual design of the Canis Compressed Air Engine. This paper brings out the mathematical design of the Canis Compressed Air engine. It also highlights the study of the kinematic and internal dynamic formula. With the engine components movement likened to an eccentric circular cam, with a radial movement $\left(\mathrm{R}_{\mathrm{i}}\right)$ and a normal movement component $\left(\mathrm{D}_{\mathrm{i}}\right)$ and a different $\left(\mathrm{d}_{\mathrm{i}}\right)$, with the magnitude of the radial movement $\left(\mathrm{R}_{\mathrm{i}}\right)$ and different $\left(\mathrm{d}_{\mathrm{i}}\right)$ considered constant, respectively 4units and 1units, the mathematical formula describing the displacement, velocity and acceleration were verified. This verification, done graphically, ended up with the conclusion that this design of a compressed air engine, complies theoretically with the recommendations of accurate intake and exhaust positioning, smooth running and high efficiency due to absence of backlashing forces during intake. The theoretical internal dynamics analysis further proofs the susceptibility of a perfect intake then explosive expansion and a perfect exhaust for every expansion chamber.
\end{abstract}

Keywords: Kinematics, dynamics, eccentric, circular, cam and free-body

\section{Introduction}

The Canis Compressed Air engine is an engine design that is powered solely by compressed air. This engine uses the rotary engine principle and prior to this paper the engine's physical appearance was designed with the geometry of the parts and their assembly established by this same author (Fru, 2015). This paper analyses the kinematics and dynamics formulations which were graphically studied and conclusions derived on the functioning principles of the machine.

\section{Kinematics Formulations of the Canis Compressed - Air (Cc) Engine}

In order to realize the kinematics formulations of this mechanism, (Vinogradov, 2000) a skeleton representation of what was thought of as the mechanism was established.

\subsection{Skeleton Display of CC Engine}

The diagram below represents what was considered as an instant in the movement of the CC engine. There too, has been associated the general axes (x,y) and the local axes ( $\left.\mathrm{x}_{1}, \mathrm{y}_{1}\right)$ of the assembly. (Kovacevic, 2009).

The graphical display brought in some satisfaction on the balancing of the mechanism, for vividly can be seen an annulment of the radial forces and even the radial movements. A complete functional cycle of this design consists of an intake, power (expansion) and exhaust. These were considered to occur for each complete wing cycle of all the expansion chambers and so, to result each to a complete cycle of the output rotor centered at the point ' $\mathrm{O}$ '.

It was noted that; $\mathrm{EC}_{5}$ (Expansion Chamber 5) was at the verge of completing its exhaust thus its wing had little or no influence on the movement of the cylinder, instead, the radial force from $\mathrm{EC}_{2}$ was compelled to be compensated by the sum of contributions donated by the other normal forces from the different ECs that were not actually in line with their respective radial reaction forces. The annulment of radial forces in this manner insinuates equilibrium in the engine design. 


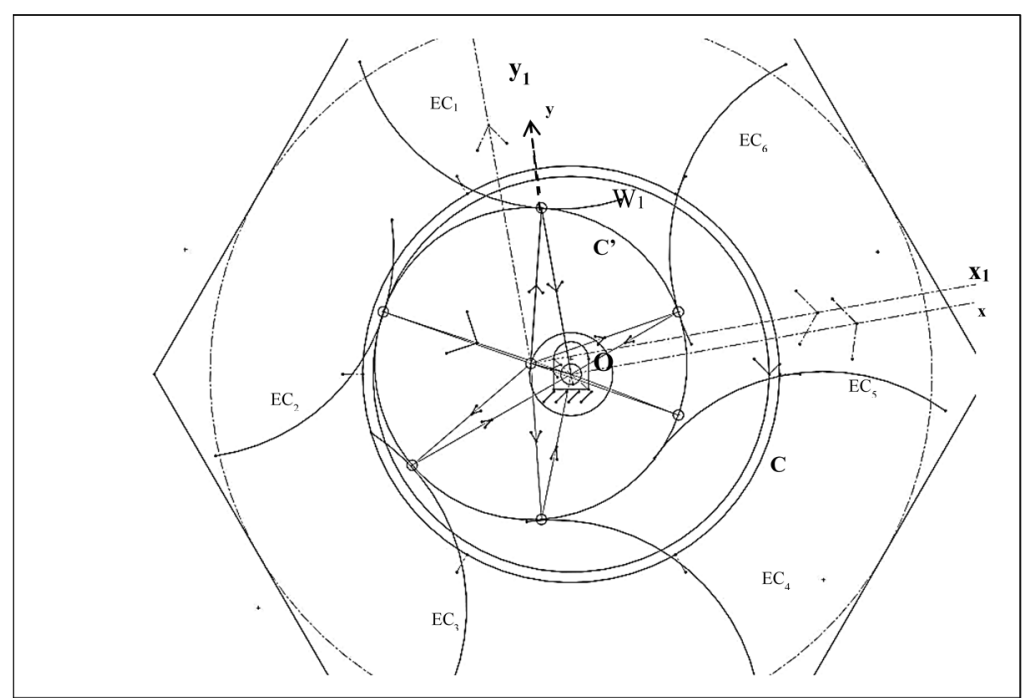

Figure 1. Skeleton display of CC engine

\subsection{Kinematic Diagram}

The movement of contact points of each wing with the circle (C') was taken for a vector triangle consisting of a radial movement $\left(\mathrm{R}_{\mathrm{i}}\right)$ and a normal movement component $\left(\mathrm{D}_{\mathrm{i}}\right)$ and a different $\left(\mathrm{d}_{\mathrm{i}}\right)$.

From prior knowledge on the $\mathrm{CC}$ engine and the above skeleton, the $\mathrm{CC}$ engine rotor cylinder was seen to experience an eccentric circular cam movement centered at ' $\mathrm{O}$ ' and tangent to the circle ' $\mathrm{C}$ ' all displayed above. This implies that the contact point of each wing with this cam experiences movements which were likened to the loop vector below.

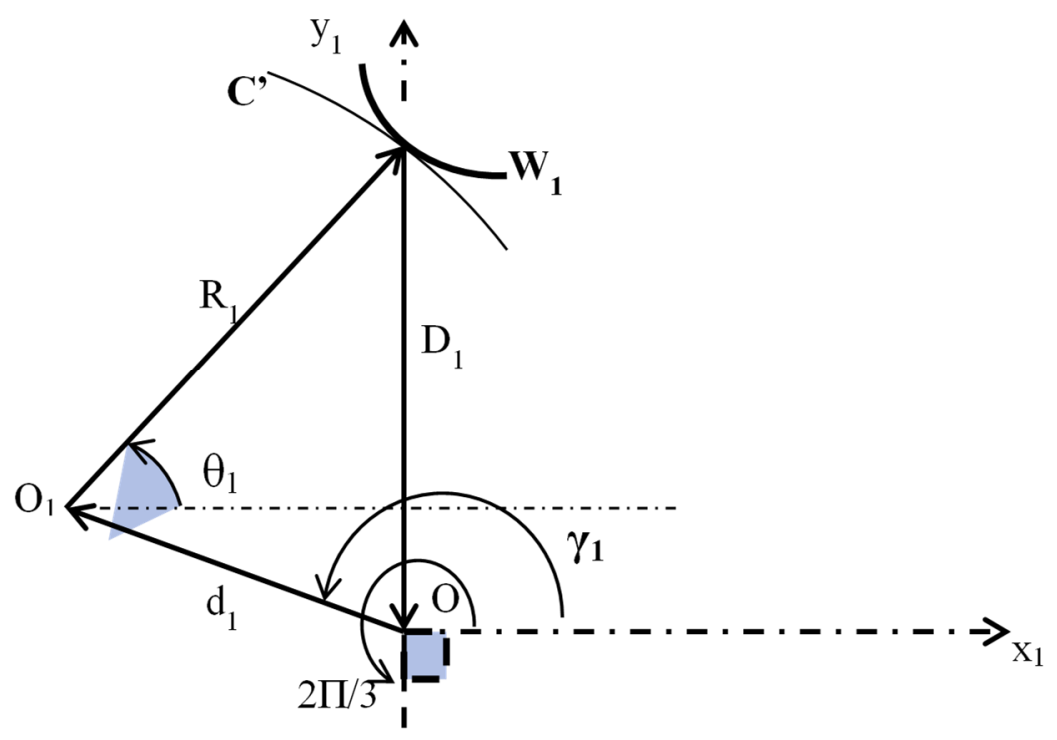

Figure 2. The links of CC engine

The above vector display was then interpreted as follows;

- $\quad \mathrm{D}_{1}=\mathrm{D}_{1}(\mathrm{t})$ that is, the magnitude of $\mathrm{D}_{1}$ varies with time but was considered as unknown

- $\mathrm{d}_{1}=$ const that is, the magnitude of $\mathrm{d}_{1}$ is constant with time

- $\mathrm{R}_{1}=$ const that is, the magnitude of $\mathrm{R}_{1}$ is constant with time

- $\theta_{1}=\theta_{1}(\mathrm{t})$ that is, the angle $\theta_{1}$ varies with time

- $\gamma_{1}=\gamma_{1}(\mathrm{t})$ that is, the angle $\gamma_{1}$ varies with time but was considered as known.

- It was equally considered that the vector $\mathrm{D}_{1}$ remains normal to our local $\mathrm{x}_{1}$-axis and equals the sum of the vectors $\mathrm{d}_{1}$ and $\mathrm{R}_{1}$. 


\subsection{Displacement Calculations}

With these interpretations, the loop closure equation was established to be equivalent to

Equation 1:

$$
R_{1}\left(\cos \theta_{1}, \sin \theta_{1}\right)+d_{1}\left(\cos \gamma_{1}, \sin \gamma_{1}\right)+D_{1}(\cos 3 \pi / 2, \sin 3 \pi / 2)=0
$$

This is an equation with two unknowns $D_{1}$ and $\theta_{1}$ which was solved as follows;

$$
\Rightarrow \mathrm{R}_{1}\left(\cos \theta_{1}, \sin \theta_{1}\right)+\mathrm{D}_{1}(\cos 3 \pi / 2, \sin 3 \pi / 2)=-\mathrm{d}_{1}\left(\cos \gamma_{1}, \sin \gamma_{1}\right)
$$

Suppose $(\cos 3 \pi / 2, \sin 3 \pi / 2)$ and $(-\sin 3 \pi / 2, \cos 3 \pi / 2)$ are unit vectors parallel and perpendicular respectively to the vector $D_{1}$. A scalar product, all through, with each of these unit vectors was carried out on (1). For $(\cos 3 \pi / 2, \sin 3 \pi / 2)$

$$
\begin{gathered}
\Leftrightarrow R_{1}\left(\cos \theta_{1}, \sin \theta_{1}\right) \cdot(\cos 3 \pi / 2, \sin 3 \pi / 2)+D_{1}=-d_{1}\left(\cos \gamma_{1}, \sin \gamma_{1}\right) \cdot(\cos 3 \pi / 2, \sin 3 \pi / 2) \\
\Rightarrow D_{1}+R_{1}\left(\cos \theta_{1} \cos 3 \pi / 2+\sin \theta_{1} \sin 3 \pi / 2\right)=-d_{1}\left(\cos \gamma_{1} \cos 3 \pi / 2+\sin \gamma_{1} \sin 3 \pi / 2\right)
\end{gathered}
$$

Equation 2:

$$
\Rightarrow D_{1}+R_{1} \cos \left(\theta_{1}-3 \pi / 2\right)=-d_{1} \cos \left(\gamma_{1}-3 \pi / 2\right)
$$

And for $(-\sin 3 \pi / 2, \cos 3 \pi / 2)$

$$
\begin{gathered}
\Leftrightarrow R_{1}\left(\cos \theta_{1}, \sin \theta_{1}\right) \cdot(-\sin 3 \pi / 2, \cos 3 \pi / 2)=-d_{1}\left(\cos \gamma_{1}, \sin \gamma_{1}\right) \cdot(-\sin 3 \pi / 2, \cos 3 \pi / 2) \\
\Rightarrow R_{1}\left(\sin \theta_{1} \cos 3 \pi / 2-\cos \theta_{1} \sin 3 \pi / 2\right)=-d_{1}\left(\sin \gamma_{1} \cos 3 \pi / 2-\cos \gamma_{1} \sin 3 \pi / 2\right)
\end{gathered}
$$

Equation 3:

$$
\Rightarrow R_{1} \sin \left(\theta_{1}-3 \pi / 2\right)=-d_{1} \sin \left(\gamma_{1}-3 \pi / 2\right)
$$

From equation (2) and (3), was deduced

$$
R_{1} \cos \left(\theta_{1}-3 \pi / 2\right)=-d_{1} \cos \left(\gamma_{1}-3 \pi / 2\right)-D_{1}
$$

And

$$
R_{1} \sin \left(\theta_{1}-3 \pi / 2\right)=-d_{1} \sin \left(\gamma_{1}-3 \pi / 2\right)
$$

Thus

$$
\begin{aligned}
\mathbf{R}_{1}{ }^{2} & =\left(d_{1} \cos \left(\gamma_{1}-3 \pi / 2\right)+D_{1}\right)^{2}+\left(d_{1} \sin \left(\gamma_{1}-3 \pi / 2\right)\right)^{2} \\
& \Rightarrow D_{1}{ }^{2}+2 D_{1} d_{1} \cos \left(\gamma_{1}-3 \pi / 2\right)+d_{1}{ }^{2}-R_{1}{ }^{2}=0
\end{aligned}
$$

Equation 4:

$$
\therefore D_{1}=-d_{1} \cos \left(\gamma_{1}-3 \pi / 2\right) \pm \sqrt{d_{1}{ }^{2} \cos ^{2}\left(\gamma_{1}-3 \pi / 2\right)+R_{1}{ }^{2}-d_{1}{ }^{2}}
$$

Given that $\mathbf{D}_{\mathbf{1}}$ is always positive therefore;

$$
\begin{gathered}
D_{1}=-d_{1} \cos \left(\gamma_{1}-3 \pi / 2\right)+\sqrt{d_{1}{ }^{2} \cos ^{2}\left(\gamma_{1}-3 \pi / 2\right)+R_{1}{ }^{2}-d_{1}{ }^{2}} \\
\therefore D_{1}=d_{1} \sin \gamma_{1}+\sqrt{R_{1}{ }^{2}-d_{1}{ }^{2} \cos ^{2} \gamma_{1}}
\end{gathered}
$$

Equation 5:

$$
\begin{gathered}
\theta_{1}{ }^{*}=3 \pi / 2+\sin ^{-1}\left(-\mathrm{d}_{1} \sin \left(\gamma_{1}-3 \pi / 2\right) / \mathrm{R}_{1}\right) \\
\therefore \theta_{1}{ }^{*}=3 \pi / 2+\sin ^{-1}\left(-\mathrm{d}_{1} \cos \gamma_{1} / \mathrm{R}_{1}\right) \\
\theta_{1}=\left\{\begin{array}{l}
\theta_{1}{ }^{*} \text { if }-\sin \theta_{1} \geq 0 \text { and } \cos \theta_{1}<0 \\
\pi-\theta_{1}{ }^{*} \text { if }-\sin \theta_{1}<0 \text { and } \cos \theta_{1}<0 \\
\pi+\theta_{1}{ }^{*} \text { if }-\sin \theta_{1}<0 \text { and } \cos \theta_{1} \geq 0 \\
2 \pi-\theta_{1}{ }^{*} \text { if }-\sin \theta_{1} \geq 0 \text { and } \cos \theta_{1} \geq 0
\end{array}\right.
\end{gathered}
$$


Given that actual dimensions of $d_{1}$ and $R_{1}$ were not yet found, it was supposed that, their respective values were 1 and 4 units respectively, then graphically; the values of $D_{1}$ for varying $\gamma_{1}$ and that of $\theta_{1}$ in radians for varying $\gamma_{1}$ were seen to follow the patterns below.

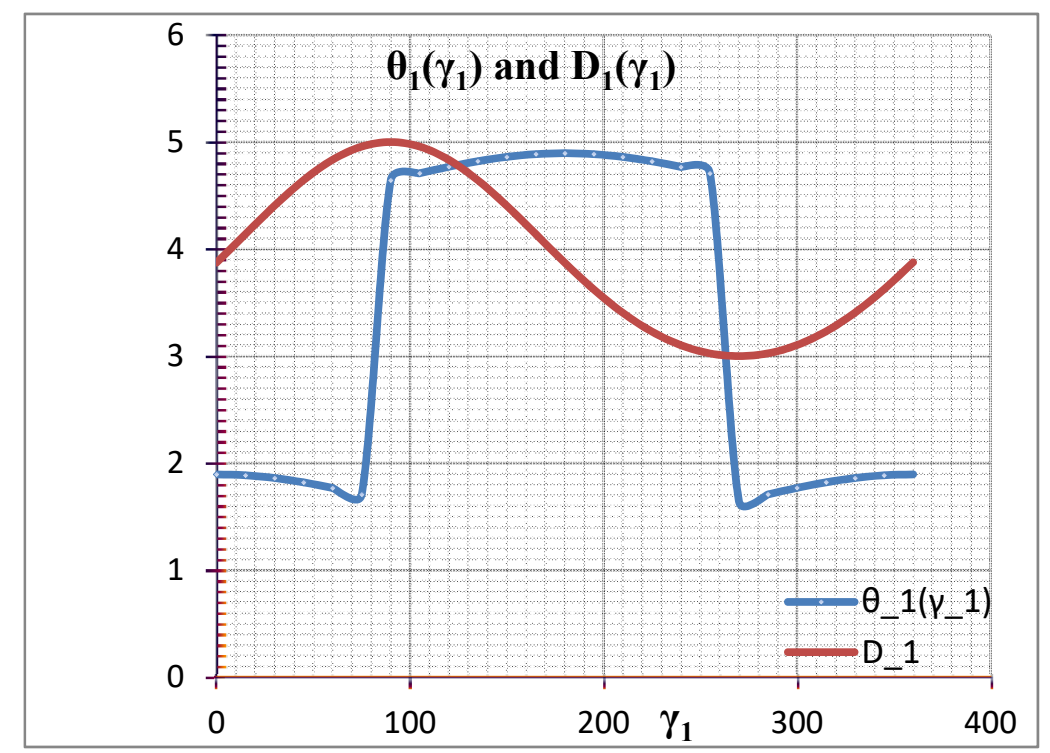

Figure 3. Displacement diagram of $\mathrm{CC}$ engine

The displacement of $\mathrm{D}_{1}$ is sinusoidal, describing a complete circle in a $360^{\circ}$ rotation of $\gamma_{1}$. The dwelling of $\theta_{1}$, during the transitions from the maximum displacement to minimum displacement describing the quasi instantaneous rise or fall, implies that time is given for the gas to expand and the wings to return to their initial position.

Further study on the velocity of the core moving components of the engine was done.

\subsection{Velocity Calculations}

From the loop closure equation above, the following velocity equation was deduced;

Equation 6:

$$
R_{1} \dot{\theta}_{1}\left(-\sin \theta_{1}, \cos \theta_{1}\right)+d_{1} \dot{\gamma}_{1}\left(-\sin \gamma_{1}, \cos \gamma_{1}\right)+\dot{D}_{1}(\cos 3 \pi / 2, \sin 3 \pi / 2)=0
$$

It was assumed that $\dot{\gamma_{1}}$ was known, for it is angular velocity expected from the rotor and that, $\dot{\mathrm{D}}_{1}$ and $\dot{\theta}_{1}$ were to be calculated. Using the unit vectors that were given above, scalar products across equation 6 were done:

For $(\cos 3 \pi / 2, \sin 3 \pi / 2)$

$$
R_{1} \dot{\theta}_{1}\left(\cos \theta_{1} \sin 3 \pi / 2-\sin \theta_{1} \cos 3 \pi / 2\right)=-d_{1} \dot{\gamma}_{1}\left(\cos \gamma_{1} \sin 3 \pi / 2-\sin \gamma_{1} \cos 3 \pi / 2\right)-\dot{D}_{1}
$$

Equation 7:

$$
\Rightarrow R_{1} \dot{\theta}_{1} \cos \theta_{1}=\dot{D}_{1}-d_{1} \dot{\gamma}_{1} \cos \gamma_{1}
$$

And for $(-\sin 3 \pi / 2, \cos 3 \pi / 2)$

$$
R_{1} \dot{\theta}_{1}\left(\sin \theta_{1} \sin 3 \pi / 2+\cos \theta_{1} \cos 3 \pi / 2\right)=-d_{1} \dot{\gamma}_{1}\left(\sin \gamma_{1} \sin 3 \pi / 2+\cos \gamma_{1} \cos 3 \pi / 2\right)
$$

Equation 8:

$$
\Rightarrow R_{1} \dot{\theta}_{1} \sin \theta_{1}=-d_{1} \dot{\gamma}_{1} \sin \gamma_{1}
$$

From equations 7 and 8, was deduced

Equation 9:

$$
\dot{\theta}_{1}=\frac{-d_{1} \dot{\gamma}_{1} \sin \gamma_{1}}{R_{1} \sin \theta_{1}}
$$


And

$$
\begin{gathered}
\dot{\mathrm{D}}_{1}=\mathbf{R}_{1} \dot{\theta}_{1} \cos \theta_{1}+\mathrm{d}_{1} \dot{\gamma_{1}} \cos \gamma_{1} \\
\Rightarrow \dot{\mathrm{D}}_{1}=\frac{\mathbf{R}_{1} \mathrm{~d}_{1} \dot{\gamma}_{1} \sin \theta_{1} \cos \gamma_{1}-\mathbf{R}_{1} \mathrm{~d}_{1} \dot{\gamma_{1}} \sin \gamma_{1} \cos \theta_{1}}{\mathbf{R}_{1} \sin \theta_{1}} \\
\Rightarrow \dot{\mathrm{D}}_{1}=\frac{\mathrm{d}_{1} \dot{\gamma_{1}} \sin \left(\theta_{1}-\gamma_{1}\right)}{\sin \theta_{1}}
\end{gathered}
$$

Equation 10:

$$
\dot{\mathrm{D}}_{1}=\frac{\mathrm{d}_{1} \dot{\gamma}_{1} \sin \left(\theta_{1}-\gamma_{1}\right)}{\sin \theta_{1}}
$$

$\dot{\gamma_{1}}$ was taken to be $\mathbf{3 0 0 0} \mathbf{r e v} / \mathbf{m i n u t e}$, the variation of $\dot{\mathbf{D}}_{\mathbf{1}}$ and $\dot{\boldsymbol{\theta}}_{\mathbf{1}}$ was then described using the graphs below;

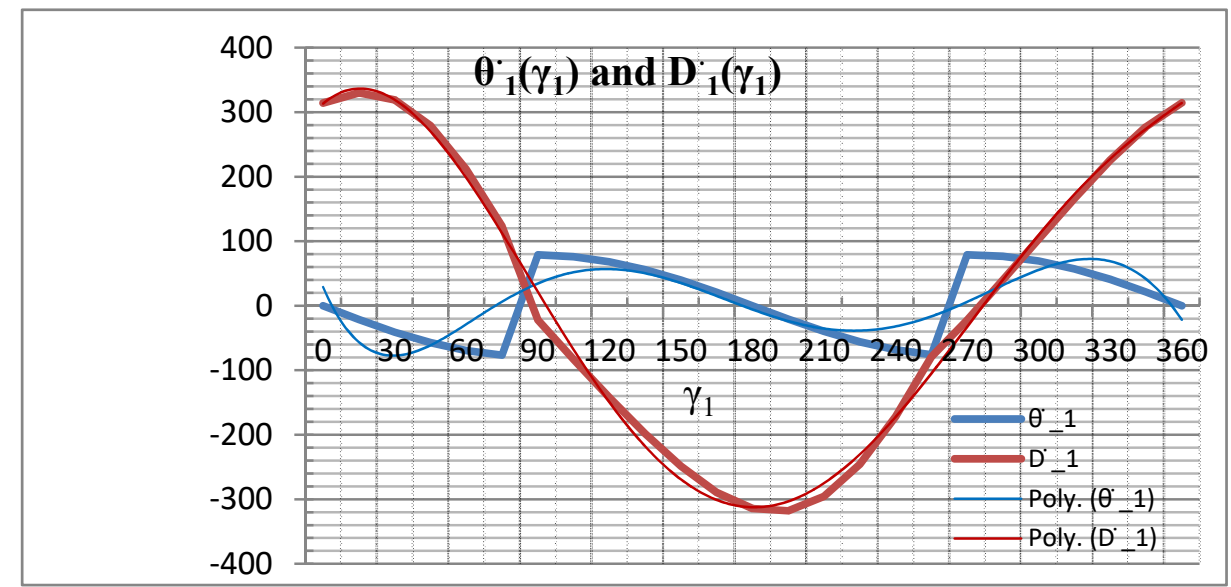

Figure 4. Velocity diagram of CC Engine

From the curve and their $6^{\text {th }}$ order polynomial trendlines above, it was observed that at the extreme displacements, the velocities were not zero instead the zero mark appears at a very brief instance. This falls in line with the requirements for a smooth exhaust and intake, thus a smooth running of the engine.

\subsection{Acceleration Calculations}

With the information given above, a study of how the different core movable components accelerate at a supposed rotational speed of the rotor, that is, $3000 \mathrm{rev/minute,} \mathrm{was} \mathrm{carried} \mathrm{out.} \mathrm{From} \mathrm{the} \mathrm{loop} \mathrm{closure} \mathrm{equation,} \mathrm{was} \mathrm{deduced}$ the following;

Equation 11:

$$
R_{1} \ddot{\theta}_{1}\left(-\sin \theta_{1}, \cos \theta_{1}\right)+\ddot{D}_{1}(\cos 3 \pi / 2, \sin 3 \pi / 2)=R_{1} \dot{\theta}_{1}{ }^{2}\left(\cos \theta_{1}, \sin \theta_{1}\right)+d_{1}{\dot{\gamma_{1}}}^{2}\left(\cos \gamma_{1}, \sin \gamma_{1}\right)
$$

Using the unit vectors quoted earlier, the scalar products with (11) deduced;

For $(\cos 3 \pi / 2, \sin 3 \pi / 2)$ :

$R_{1} \ddot{\theta}_{1}\left(-\sin \theta_{1} \cos 3 \pi / 2+\cos \theta_{1} \sin 3 \pi / 2\right)+\ddot{D}_{1}$

$$
=R_{1} \dot{\theta}_{1}{ }^{2}\left(\cos \theta_{1} \cos 3 \pi / 2+\sin \theta_{1} \sin 3 \pi / 2\right)+d_{1}{\dot{\gamma_{1}}}^{2}\left(\cos \gamma_{1} \cos 3 \pi / 2+\sin \gamma_{1} \sin 3 \pi / 2\right)
$$

Equation 12:

$$
\Rightarrow \ddot{D}_{1}-R_{1} \ddot{\theta}_{1} \cos \theta_{1}=-R_{1} \dot{\theta}_{1}^{2} \sin \theta_{1}-d_{1}{\dot{\gamma_{1}}}^{2} \sin \gamma_{1}
$$

And for $(-\sin 3 \pi / 2, \cos 3 \pi / 2)$

$$
\begin{aligned}
\mathbf{R}_{1} \ddot{\theta}_{1}\left(\sin \theta_{1} \sin 3 \pi / 2+\cos \theta_{1} \cos 3 \pi / 2\right) \\
=\mathbf{R}_{1} \dot{\theta}_{1}{ }^{2}\left(-\cos \theta_{1} \sin 3 \pi / 2+\sin \theta_{1} \cos 3 \pi / 2\right) \\
+d_{1}{\dot{\gamma_{1}}}^{2}\left(-\cos \gamma_{1} \sin 3 \pi / 2+\sin \gamma_{1} \cos 3 \pi / 2\right)
\end{aligned}
$$


Equation 13:

$$
\Rightarrow-\mathbf{R}_{1} \ddot{\theta}_{1} \sin \theta_{1}=\mathbf{R}_{1} \dot{\theta}_{1}{ }^{2} \cos \theta_{1}+d_{1}{\dot{\gamma_{1}}}^{2} \cos \gamma_{1}
$$

From equations 12 and 13, we can result to;

Equation 14:

$$
\ddot{\theta}_{1}=\frac{-R_{1} \dot{\theta}_{1}{ }^{2} \cos \theta_{1}-d_{1}{\dot{\gamma_{1}}}^{2} \cos \gamma_{1}}{R_{1} \sin \theta_{1}}
$$

And

$$
\begin{gathered}
\ddot{D}_{1}=\mathbf{R}_{1} \ddot{\theta}_{1} \cos \theta_{1}-\mathbf{R}_{1} \dot{\theta}_{1}{ }^{2} \sin \theta_{1}-d_{1} \dot{\gamma}_{1}{ }^{2} \sin \gamma_{1} \\
\Rightarrow \ddot{D}_{1}=R_{1} \cos \theta_{1}\left(\frac{-R_{1} \dot{\theta}_{1}{ }^{2} \cos \theta_{1}-d_{1}{\dot{\gamma_{1}}}^{2} \cos \gamma_{1}}{R_{1} \sin \theta_{1}}\right)-R_{1} \dot{\theta}_{1}{ }^{2} \sin \theta_{1}-d_{1} \dot{\gamma}_{1}{ }^{2} \sin \gamma_{1} \\
\Rightarrow \ddot{D}_{1}=\frac{-R_{1} \dot{\theta}_{1}{ }^{2} \cos ^{2} \theta_{1}-d_{1}{\dot{\gamma_{1}}}^{2} \cos \gamma_{1} \cos \theta_{1}-R_{1} \dot{\theta}_{1}{ }^{2} \sin ^{2} \theta_{1}-d_{1} \dot{\gamma}_{1}{ }^{2} \sin \gamma_{1} \sin \theta_{1}}{\sin \theta_{1}}
\end{gathered}
$$

Equation 15:

$$
\Rightarrow \ddot{D}_{1}=\frac{-R_{1} \dot{\theta}_{1}{ }^{2}-d_{1}{\dot{\gamma_{1}}}^{2} \cos \left(\gamma_{1}-\theta_{1}\right)}{\sin \theta_{1}}
$$

A graphical display of $\ddot{\boldsymbol{\theta}}_{\mathbf{1}}$ and $\ddot{\mathbf{D}}_{\mathbf{1}}$ can be perceived below;

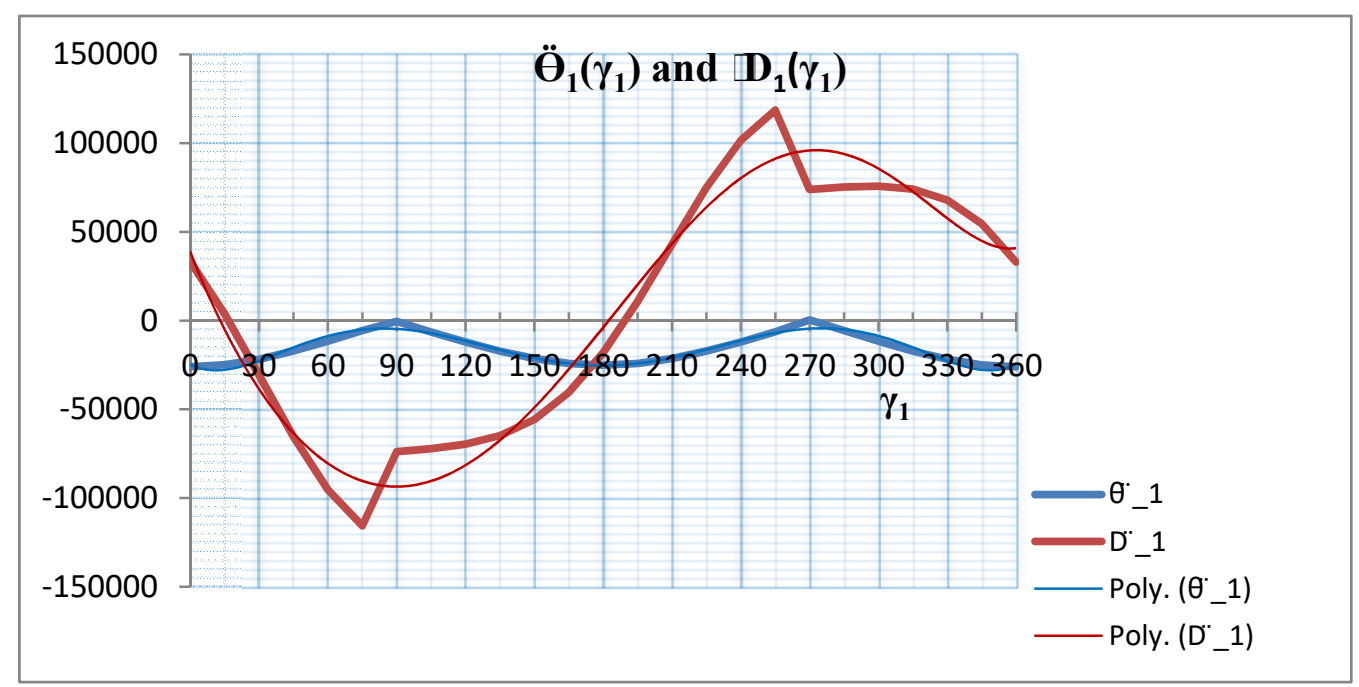

Figure 5. Acceleration diagram of CC Engine

The above graphical presentation of $\ddot{D}_{1}$ its respective $6^{\text {th }}$ order polynomial trendline proof that shortly before the intake, that is, when $\mathrm{D}_{1}$ is at its maximum, the cam would have decelerated enough so that by the start of the expansion, there is very little or no resistance due to further displacement in the reactive direction and so an efficient use of the potential energy stored in the compressed air. $\ddot{\theta}_{1}$ shows a steady angular deceleration whose peak (when it touches the horizontal axis) is attained during the intake and exhaust. With our knowledge so far on the kinematics of the CC engine, we can see that the minimum pressure angle of our mechanism is zero.

Vividly these graphical representations brought in some satisfaction on the kinematics of the engine, to be better convinced with the functioning of CC engine, the dynamic formulations of the engine was done. (Psanis, 2007)

\section{Internal Dynamics Formulations of the Canis Compressed Air (CC Engine) Engine}

\subsection{Skeleton of Dynamic Forces CC Engine}

Consider figure 1, the links acting at the wing contact base of expansion chamber 1 (EC1) were isolated to give the following skeleton diagram (it was assumed the masses were insignificant). 


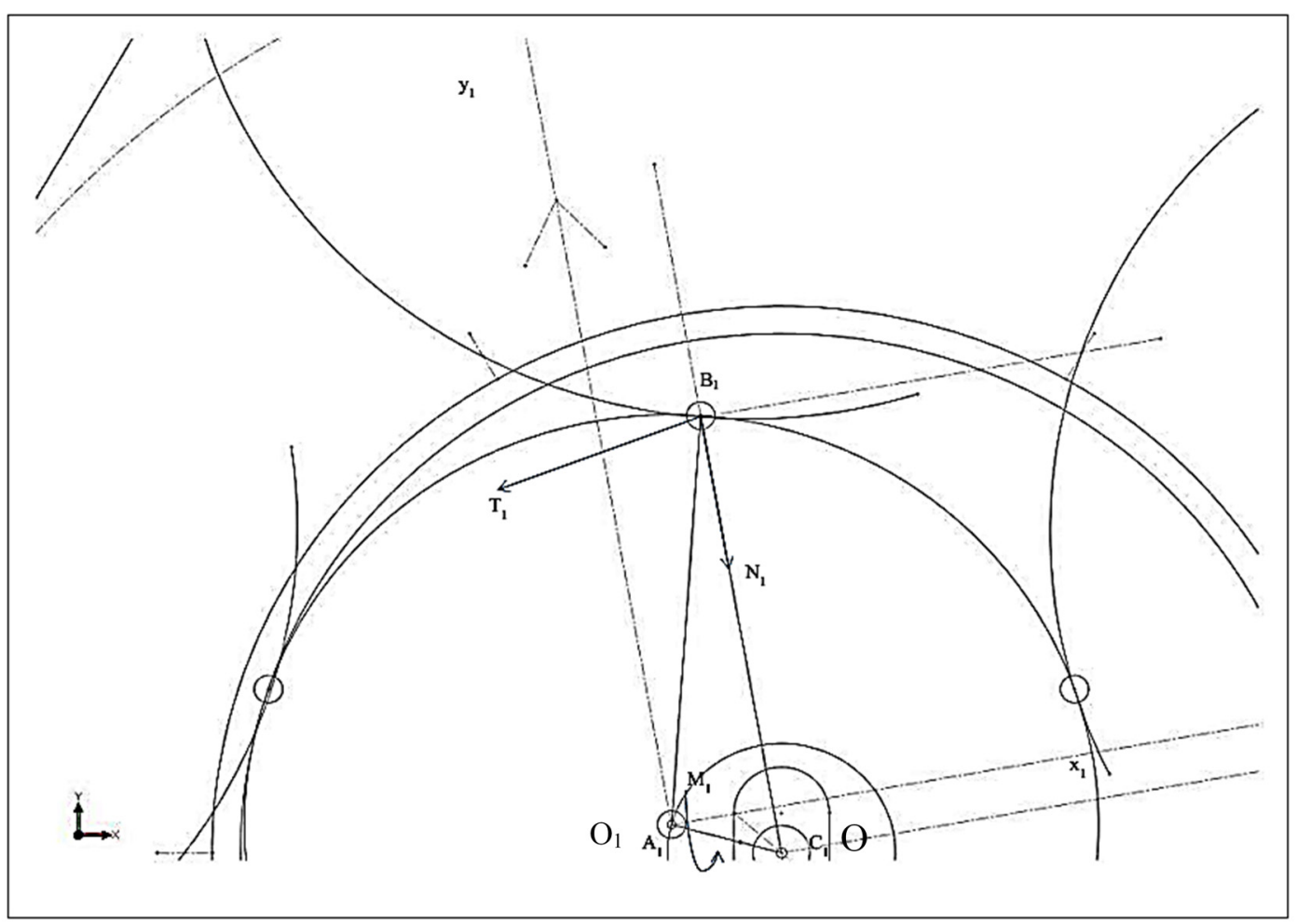

Figure 6. Skeleton of $\mathrm{CC}$ engine with dynamic forces

\subsection{Free Body Diagram of CC Engine}

The free body diagram of the $\mathrm{CC}$ engine was established as can be seen below. For each link, the local unit vector tangent and normal were represented by $\xi$ and $\eta$. $\mathrm{R}_{1 \mathrm{D}}$ represented an unknown resultant and $\mathrm{M}_{1}$ a known torque; the expected torque of the engine.

\subsection{Dynamic Formulation}

Link $d_{1}$;

$$
F_{1 d}-R_{1 d}=0
$$

And

$$
d_{1} \times F_{1 d}+M_{1}=0
$$

Thus

$$
d_{1} F_{1 d} \sin \left(\theta_{1}-\gamma_{1}\right)+M_{1}=0
$$

Equation 16:

$$
F_{1 d}=\frac{-M_{1}}{d_{1} \sin \left(\theta_{1}-\gamma_{1}\right)}
$$

From the free body diagram, it was deduced that;

Equation 17:

$$
F_{1 d}=R_{1 R}=-R_{1 d}=-R_{1 D}=-F_{1 R}
$$

Link $D_{l}$ :

$$
\begin{gathered}
R_{1 D}+T_{1 D}+N_{1 D}=0 \\
R_{1 D}\left(\cos \theta_{1}, \sin \theta_{1}\right)^{T}+T_{1 D}(-1,0)^{T}+N_{1 D}(0,-1)^{T}=0
\end{gathered}
$$

Thus

Equation 18:

$$
T_{1 D}=R_{1 D} \cos \theta_{1}
$$


And

$$
N_{1 D}=R_{1 D} \sin \theta_{1}
$$

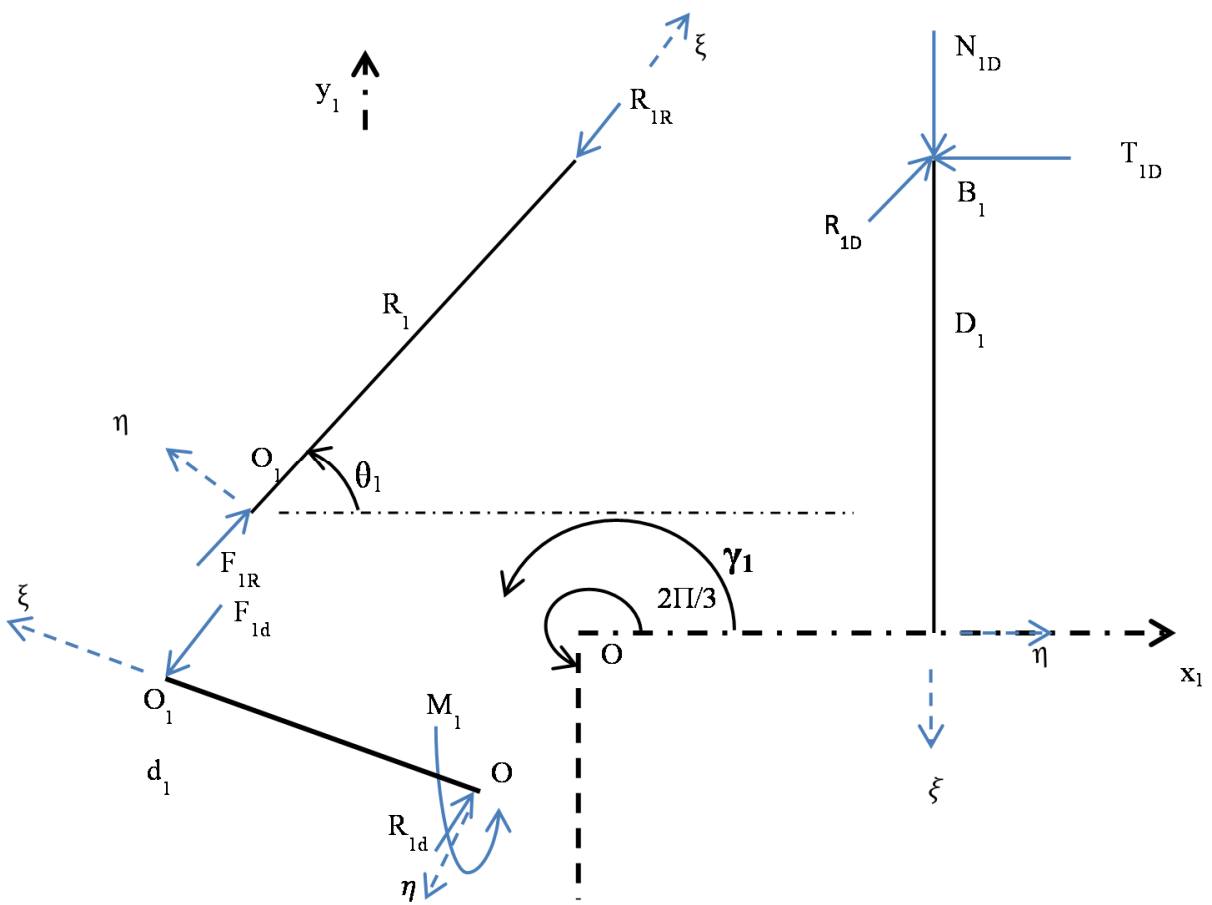

Figure 7. Free body diagram of $\mathrm{CC}$ engine

With a rotor torque of 20 units, a graphical study of the forces was done as can be seen below.

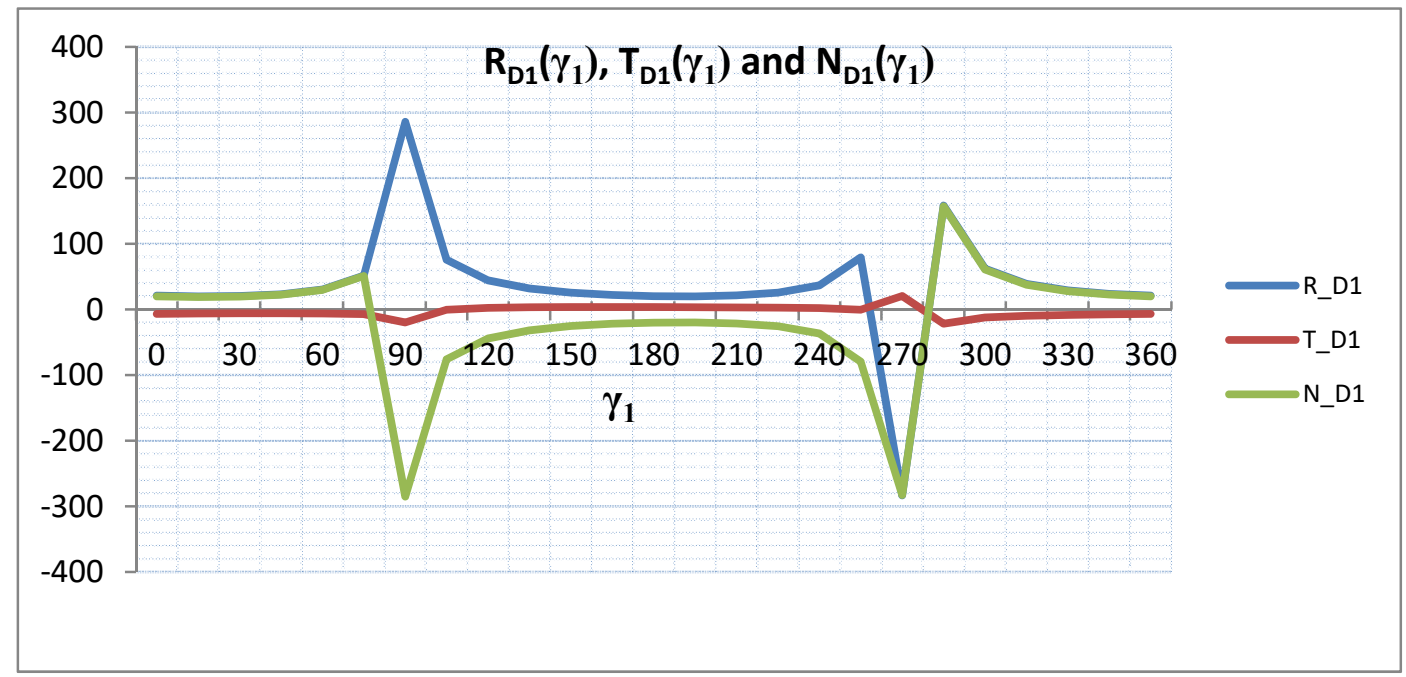

Figure 8. Dynamic forces of CC Engine

The peak of the resultant force shortly after the intake and its trough at the exhaust brought about the conclusion that the system will react readily according to expectation, producing the maximum rotatory force per expansion chamber, during the intake. This will permit the mechanism to overcome forces of inertia. The mathematical analysis show that the resultant force gets to about ten times the average shortly after the intake and it drops down by similar figures during the exhaust. 


\section{Conclusion}

This paper concludes with the study of the internal dynamic forces which are governed by the action - reaction principles. The findings at this level further concretize the susceptibility of a perfect intake, explosive expansion and smooth exhaust. With this much understanding of the system design, the smooth functioning of this engine is assured theoretically with little or no conflicting forces and movements. The next step will have to deal with the external forces which will yield the appropriate torque and speed of the engine thus a step closer to dimensioning the engine component parts.

\section{References}

Fru, N. T. (2015). Design and Simulation of a compressed air Engine. Bambili, N.W, Cameroon: University of Bamenda.

Kovacevic, P. A. (2009). Principles of Mechanical Design. School of Engineering and Mathematical Sciences. UK: Engineering Design Council, UK.

Psanis, C. (2007). Modelling and Experimentation on Air Hybrid Engine Concepts for Automotive Applications. West London, United Kingdom: Brunel University.

Vinogradov, O. (2000). Fundamentals of Kinematics and Dynamics of Machines and Mechanisms. Boca Raton, Florida: CRC Press LLC, 2000 N.W. Corporate Blvd.

\section{Copyrights}

Copyright for this article is retained by the author(s), with first publication rights granted to the journal.

This is an open-access article distributed under the terms and conditions of the Creative Commons Attribution license (http://creativecommons.org/licenses/by/4.0/). 